\title{
A New Convolutional Neural Network based on a Saprse Convolutional Layer for Animal Face Detection
}

\author{
Islem Jarraya ${ }^{\mathrm{a}, *}$, Fatma BenSaid ${ }^{\mathrm{a}}$, Wael Ouarda ${ }^{\mathrm{a}, \mathrm{b}}$, Umapada Pal ${ }^{\mathrm{c}}$, Adel M. \\ Alimi $^{\mathrm{a}, \mathrm{d}}$ \\ ${ }^{a}$ REGIM-Lab.: REsearch Groups in Intelligent Machines, University of Sfax, National \\ Engineering School of Sfax (ENIS), BP 1173, Sfax, 3038, Tunisia \\ ${ }^{b}$ Digital Research Center of Sfax, B.P. 275, Sakiet Ezzit, Sfax, 3021, Tunisia \\ ${ }^{c}$ Computer Vision and Pattern Recognition Unit, Indian Statistical Institute, 203, B.T. \\ Road, Kolkata 700 108, India \\ ${ }^{d}$ Department of Electrical and Electronic Engineering Science, Faculty of Engineering and \\ the Built Environment, University of Johannesburg, South Africa
}

\begin{abstract}
This paper focuses on the face detection problem of three popular animal categories that need control such as horses, cats and dogs. To be precise, a new Convolutional Neural Network for Animal Face Detection (CNNAFD) is actually investigated using processed filters based on gradient features and applied with a new way. A new convolutional layer is proposed through a sparse feature selection method known as Automated Negotiation-based Online Feature Selection (ANOFS). CNNAFD ends by stacked fully connected layers which represent a strong classifier. The fusion of CNNAFD and MobileNetV2 constructs the new network CNNAFD-MobileNetV2 which improves the classification results and gives better detection decisions. Our work also introduces a new Tunisian Horse Detection Database (THDD). The proposed detector with the new CNNAFDMobileNetV2 network achieved an average precision equal to $99.78 \%, 99 \%$ and $98.28 \%$ for cats, dogs and horses respectively.
\end{abstract}

\footnotetext{
${ }^{\star}$ Fully documented templates are available in the elsarticle package on CTAN

${ }^{*}$ Corresponding author

Email addresses: islem.jarraya@enis.tn (Islem Jarraya),

fatma.bensaid@regim.usf.tn (Fatma BenSaid), wael.ouarda@ieee.org (Wael Ouarda), umapada@isical.ac.in (Umapada Pal), adel.alimi@regim.usf.tn (Adel M. Alimi)

$U R L:$ http://www.members.regim.org/islemjarraya (Islem Jarraya)
} 
Keywords: Animal Face Detection, MobileNetV2, Convolution Neural Network, ANOFS

\section{Introduction}

In a natural scene, face detection is a long-term objective for remote control and security needs. Ensuring safety for animals is an important task, particularly for breeders to fight against fraud and animal theft and to enforce health monitoring and traceability. Using facial features, animal monitoring does not require a direct contact with the sensor and the animal will thus be at ease. Animal face detection can be used for many security applications that identify animal faces, gender/age detection and visual monitoring [1. Regretfully, it is still too difficult to detect animal faces given that face textures and shapes are grossly diverse. This is probably the reason for the small number of approaches. The face detection task is still extremely difficult mainly because of the wide intra-class variation, illumination change, variable pose, complex background and partial occlusion. Despite these difficulties, recent research has achieved significant progress to resolve the interesting detection problems. The detection rate has reached nearly $90 \%$ of the human face using boosting-based [2] and CNN-based [3] approaches. Traditional human face detectors adopting handcrafted features have been replaced in many works by deep convolutional neural networks with the ability to extract discriminative facial features. In the literature, the detection procedure usually includes three steps: block generation (multi-scale sliding windows or region proposals), face classification (in the backbone of the detector) and post-processing (non-maximum suppression and bounding box regression). In fact, the performance of face detectors is mainly influenced by the face classification network also known as the backbone. Duan et al. 4. discovered that the detector and the classifier of the general object detection have comparable performances using the same backbone. These explain that the designed backbone for the classification dataset is applied easily to the general object detection which gets an excellent mAP (mean Average Precision) 
score. Existing detectors, especially those for humans, have already taken on known CNN architectures as backbones. Convolutional neural networks (CNNs) are strong and fascinating classification tools. This is one of the reasons why Deep Learning is immensely popular and widely used for computer vision tasks. Given the rapid development, the questions that must arise are the following: Are CNNs flawless? Are they the best? In fact, there are different challenges during $\mathrm{CNN}$ training:

- Most network optimization algorithms (such as SGDM and ADAM) use the backpropagation method to set the layer weights. Backpropagation has yielded good training results in recent years but it is not a very effective way to learn as it requires a huge dataset for CNNs [5].

- According to Geoffrey E Hinton [6, pooling layers eliminate a great deal of information and ignore the relationship between image parts. In face detectors, for instance, just combining some features (mouth, eyes, face oval and nose) makes a face.

- Face detectors use CNNs which represent a big number of parameters and layers. This leads to much training time and high computational complexity.

The new challenging issue consists in creating a new convolutional neural network that effectively exploits the animal face characterization maintaining the relationship between image parts and using the smallest number of operations and parameters without the need for a huge dataset in order to obtain a robust and fast detector. To deal with the above-listed problems, this paper introduces the Convolutional Neural Network CNNAFD merged with MobileNetV2 for the purpose of an efficient animal face detection.

\section{Related works}

The number of works in this area is very limited due to the complication of the animal face detection task. The existing related works in this field are as fol- 
lows: Zhang et al. [7] proposed a set of Haar of Oriented Gradients (HOOG) to capture the texture and shape features on the animal head (such as cats, tigers, pandas, foxes and cheetahs). Using the Cat Database, they found a precision equal to $95 \%$ and a recall equivalent to $99.8 \%$. Yamada et al. [8] proposed detecting dog and cat heads using edge-based features. They performed their method on a set of cat and dog images from the web. The recall rate was equal to $85 \%$ on the cat set and $90 \%$ on the dog set. Mukai et al. 9] focused on cat and dog face detection. They used the same Viola-Jones method and employed both the HAAR and the HOG descriptors for feature extraction. Using $58 \mathrm{im}-$ ages from the Cat Database for the test, they found a recall equal to $96.6 \%$ and a precision equivalent to $75.7 \%$. However, they achieved a recall equal to $98.3 \%$ and a precision equivalent to $90.8 \%$ using 60 images from the Stanford Dogs Dataset. Vlachynska et al. [10] used the faster R-CNN with ResNet network for dog face detection. They found an Average Precision equal to $98 \%$ on the for dog face detection noticed an Average Precision equivalent to $92 \%$ on the Columbia Dogs Dataset and the Oxford-IIIT Pet Dataset.

In the last decades, the development of facial recognition systems has been achieved using manually-noted databases in order to locate the facial area in the image. Overall, facial recognition systems have not been automated by facial detection systems [12, 13, 14]. However, these methods allow high recognition rates but their systems lack automatic face detection, which is why the animal face detection system is important to ensure safety and security.

\section{CNNAFD: Convolutional Neural Network for Animal Face Detection}

A small convolutional network and a fast training with a small database were taken into consideration in order to overcome the previously-mentioned challenges. Despite the large diversity of animal head textures, each animal species has a distinctive head form with a similar shape. Consequently, the gra- 
dient features were considered because they are invariant to photometric and geometric transformations. Furthermore, as Dalal and Triggs [15] discovered, fine orientation sampling, coarse spatial sampling and strong local photometric normalization make it possible to ignore the object movement as long as it maintains a roughly vertical position without a big transformation as is the case

90 for animal face. The gradient features could thus be suited for animal face detection in images. The Automated Negotiation-based Online Feature Selection (ANOFS) is a sparse online learning method introduced by BenSaid and Alimi [16, 17, 18. The aim of this method is to select a small number of features for binary classification on small databases and thereby replace the traditional optimizers (such as SGDM and ADAM) by the ANOFS, which decreases the number of layer parameters and operations. Moreover, using ANOFS helps to find the most expressive features and extract the best representation of the animal face by keeping the relationship between the face parts during the training. In fact, this paper presents the proposed convolutional neural network CNNAFD. As shown in Fig. 1, the CNNAFD network included five types of layers: INPUT was used to keep the raw pixel values of the image. The gradient convolution features that were connected to local regions in the input were computed by the Gradient convolution (gradient-CONV) layer. The ANOFS convolution features were computed by the ANOFS convolution (ANOFS-CONV) layer connected to the previous convolution features. A pooling layer (Non-zero POOL) was used to perform the downsampling operation along the produced ANOFS-CONV output. The scores were computed through a Fully Connected (FC) layer. In this network, the layers had the shape of vectors instead of matrices.

\subsection{Gradient-CONV layer}

110

The gradient-CONV layer incorporated some constraints and achieved some deformations using local receptive fields, gradient features and spatial subsampling. Each unit in the output vector was connected to local regions of neighborhood pixels in the input image as usual (Fig. 11). The output vector $C$ was considered as a feature map produced by a local window of size $16 * 16 * 1$ which 
scanned over the plane of the image with a stride of 8 pixels. The same principle of the HOG descriptor proposed by Triggs and Dalal [15] was applied in this layer for feature calculation. Each window was divided into four sub-windows of $8 * 8$. The gradient magnitude and the gradient angle were calculated for each sub-window in order to construct a normalized $36 * 1$ vector over the whole window. The produced gradient vector represented a unit in the output feature map of the gradient convolution layer. The proposed measures of this layer produced the best results in our experiments. In fact, the improvement was insignificant whether the window size was smaller or bigger. Following Triggs and Dalal [15, both the gradient magnitude and the gradient angle were calculated using the intensity of each pixel through the following expressions. The gradient-CONV layer extracted each feature vector automatically and then sent it to the second convolutional layer.

$$
\operatorname{Mag}(x, y)=\sqrt{f(y)^{2}+f(x)^{2}}, \theta(x, y)=\tan ^{-1}\left(\frac{f(x)}{f(y)}\right)
$$

\subsection{ANOFS-CONV layer}

Various maps (vectors) were constructed in this layer using various filters and thus relevant features could be selected. The ANOFS-CONV layer had a size equal to that of the gradient-Conv layer. Each neuron in the gradient-CONV layer had a unique relationship with the opposite neuron in the ANOFS-CONV layer, which reduced the number of parameters. The sparse ANOFS-CONV maps were produced when the ANOFS-CONV weights $W$ were multiplied by ${ }_{35}$ the gradient-CONV output $C$ with a linear activation function $(f(x)=x)$ as shown in the following equation:

$$
f\left(x_{i}\right)=C_{i} * W_{i} \text { with } i=1 . . n \text { : number of features }
$$

\subsection{Non-zero POOL layer}

Each ANOFS-CONV kernel weight contained a big number of zeros. The pooling layer summed up the sparse convolutional output by eliminating all the values corresponding to zero and keeping only the relevant features. Unlike the 


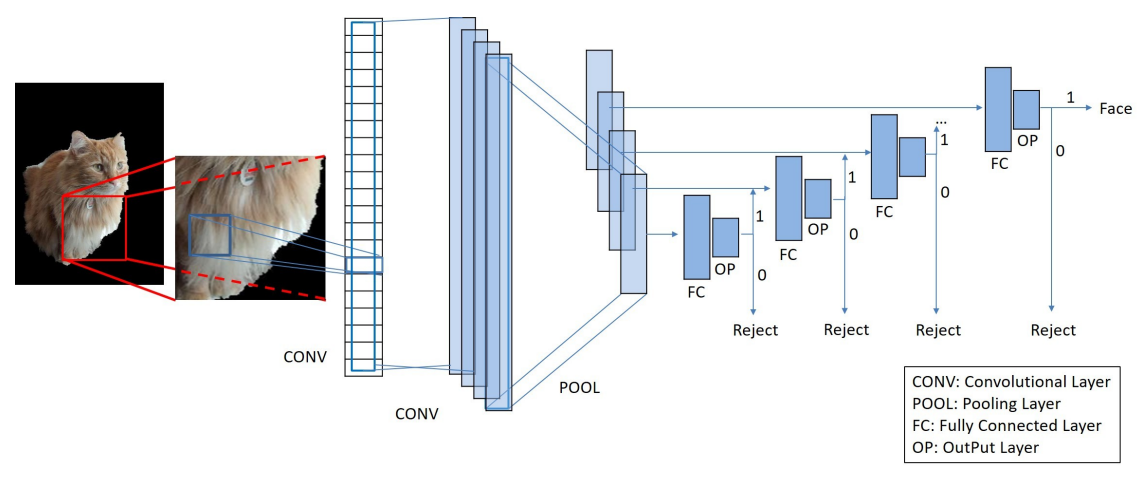

Figure 1: CNNAFD: Convolutional Neural Network for Animal Face Detection

max pooling, Non-zero POOL maintained the relationship between image parts by keeping the same arrangement of values.

\subsection{Stacked Fully Connected (FC) layers}

The proposed CNNAFD was completed with stacked Fully Connected (FC) layers for classification. Once one FC layer classified a region (Window) as nonface, the region was rejected without going through the rest of the FC layers. In fact, each FC layer was connected to a Non-zero POOL vector and ended with an OutPut layer (OP). The stacked FC layers were applied using the tangent sigmoid transfer function and the stochastic gradient descent with momentum (SGDM). Each FC layer represented a weak classifier algorithm. Nonetheless, when combining their decisions, the stacked FC layers represented a strong classifier.

\section{Proposed training methodology of CNNAFD}

The training database was divided into $n$ overlapped sub-sets. The gradientCONV vectors of all images were extracted from each sub-set to produce $n$ new sparse convolotional filters. In fact, Fig. 2 shows that each ANOFS-CONV filter was trained separately on a specific set of data using the Automated Negotiationbased Online Feature Selection (ANOFS) method. This sparse method has been accurate enough to create a weight vector for binary classification. The same 

weights were zeros) used by [16, 17, 18] were chosen for use. BenSaid et al. [16, 18, proved the effectiveness of the prediction performance of this fraction on several public large-scale benchmark datasets and thereby each pooling filter summarized the sparse ANOFS-CONV map by $10 \%$ of its real size.

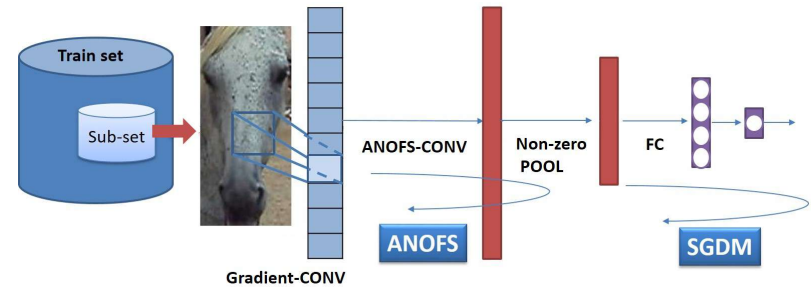

Figure 2: Training process for CNNAFD

\section{CNNAFD-MobileNetV2: fusion of CNNAFD and MobileNetV2}

For more efficiency, the addition of CNNAFD was proposed to strengthen the detection process. Table 1 presents the last CNNs which have appeared in the last 10 years. In order to keep a small number of parameters, the decisions of MobileNetV2 which presents fewer parameters were merged with CNNAFD. As shown in Fig. 3, the fusion was applied using a neuron with the tangent sigmoid transfer function and the stochastic gradient descent with momentum (SGDM). This neuron that represented the final FC layer of the network triggered the final detection decision. The detection system was based on the same YOLOv2 strategy as indicated in Fig. 4. The proposed regions were applied on the original image and used as inputs by CNNAFD. As stated above, the resulting decisions of MobileNetV2 and CNNAFD were merged by the final FC layer to obtain the final detection decision.

\section{Experimental study}

The experiments were performed on the Nvidia GeForce 920MX GPU and 180 a memory of 20 GB. 
Table 1: A comparison between CNN parameters.

\begin{tabular}{|l|l|}
\hline Network & Parameter Count \\
\hline AlexNet [19] & 60 million \\
\hline VGG-16 [20] & 138 million \\
\hline VGG19 [20] & $143,667,240$ \\
\hline GoogLeNet [2] & 5 million \\
\hline Inception-v3 [22] & $(>23$ million $)$ \\
\hline ResNet-50 [23] & 25 million \\
\hline DenseNet-190 [24] & 40 million \\
\hline MobileNetV2 [25] & 3,4 million \\
\hline CNNAFD & 1 million \\
\hline CNNAFD-MobileNetV2 & $\mathbf{4 , 4}$ million \\
\hline
\end{tabular}

\subsection{Databases}

In the experiments, the proposed CNNAFD-MobilNetV2 backbone was performed on three real-world databases:

- The THDD 11 database (Tunisian Horse Detection Datadase) included a set of horse images which were taken at different distances ranging from 1 to 2 meters relative to the horses. The collected database consisted of 703 horse images for the training and 400 images for the testing (Fig. 5). The testing set contained 415 horse faces.

- The Cat Database 26] involved 10,000 cat head photos. The photos were mainly downloaded from Flickr and were paired with data files that specified the position of each cat ears, eyes and mouth.

- The Stanford Dogs Dataset 27] included over 20,580 annotated images of 120 dog breeds. For accurate evaluation, manual face annotations were made as they did not exist for the whole face area in the Cat Database

\footnotetext{
${ }^{1}$ http://www.regim.org/publications/databases/thdd/
} 


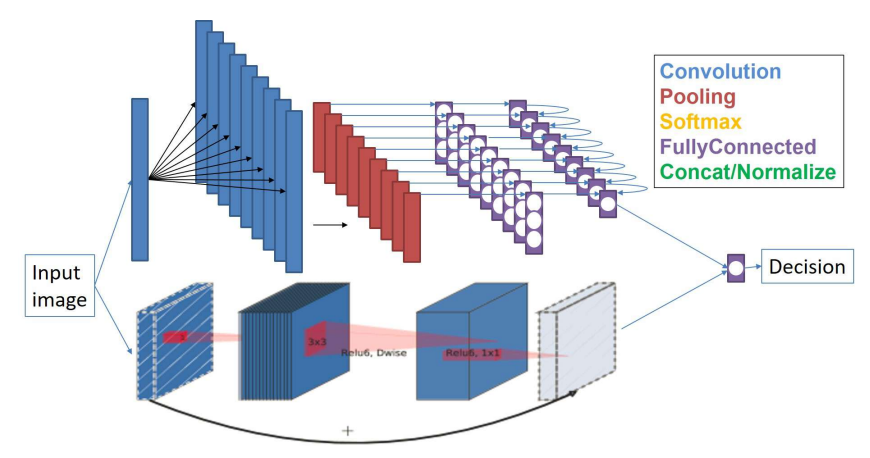

Figure 3: CNNAFD-MobileNetV2: fusion of Convolutional Neural Network for Animal Face Detection and MobileNetV2

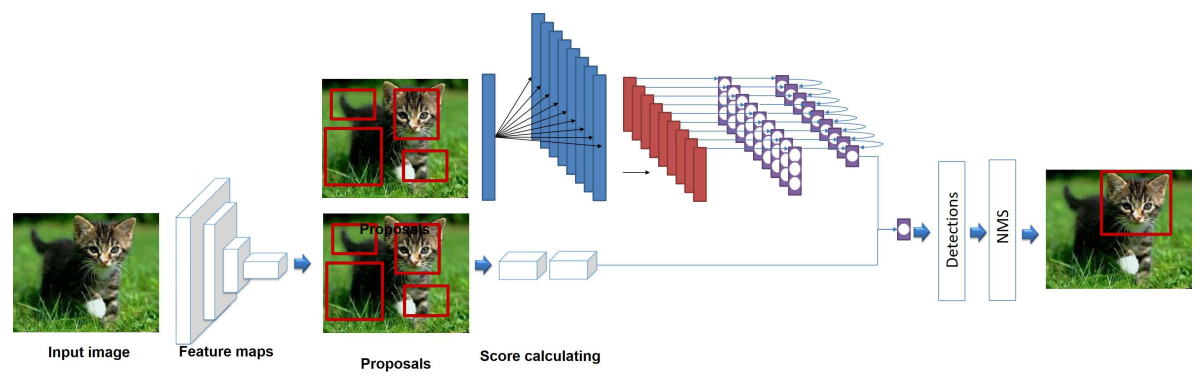

Figure 4: Proposed Detection System

and in the Stanford Dogs Dataset.

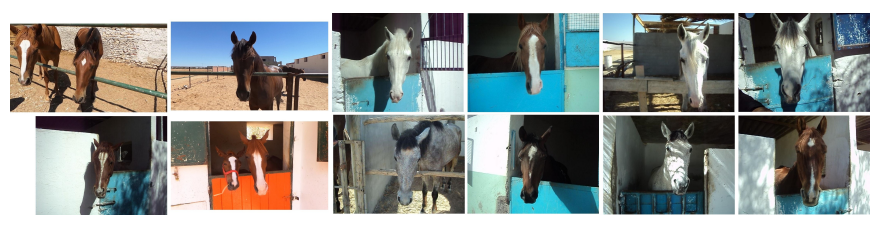

Figure 5: An illustration of capture conditions of THDD

\subsection{Experimental results}

For an accurate assessment, the proposed backbone CNNAFD-MobileNetV2 was tested on three benchmarks for animal face classification. Motivated by face classification, our work began with face proposals whereby 1124 annotated faces and 10,000 non-face samples were collected from the testing part of THDD, 3000 
annotated faces and 10,000 non-face samples were gathered from the testing part of the Cat Database as well as 1000 annotated faces and 10.000 non-face samples from the testing part of the Stanford Dogs Dataset. As a result, large-scale datasets were constructed for the evaluation of the two-class classification. Besides, different Convolutional Neural Networks were evaluated and compared. The proposed CNNAFD-MobileNetV2 and CNNAFD were compared with other CNNs such as MobileNetV2 and GoogLeNet which have represented the smallest number of parameters and ResNet-50 which is widely used for human face detection [28, 29]. A transfer learning of the four pre-trained CNNs was made on the training set of the THDD, Cat Database and Stanford Dogs Dataset. The last FC layer of these CNNs was replaced with a new FC layer having two outputs (face/non-face). As shown in table 1, the CNNAFD and CNNAFDGoogLeNet present a small number of parameters.

The field of face detection has been dominated by generic object detection methods. A slight difference was actually witnessed between the face detection and the generic object detection. Consequently, it was necessary to discuss and compare the proposed detector with object detection methods such as Faster R-CNN [30, YOLOv3 [31, SSD [32] which have also been used for human and animal face detection. The Detectron2 platform 33. for object detection has 220 become one of the most widely adopted open source projects by Facebook Artificial Intelligence Research (FAIR). The SSD MobileNetV2 is a Single-Shot multibox Detection (SSD) network designed to perform real-time object detection on mobile devices. YOLOv5 [34] is the last improved version of the YOLO detector using CSPNet 35. Thus, there was a reason for comparing our detector with Detectron2 using Faster R-CNN with ResNext-101 [33, YOLOv3-tiny with Darknet-53 11], YOLOv5 with CSPNet and SSD with MobileNetV22] presented by TensorFlow [36.

\footnotetext{
${ }^{2}$ https://blog.roboflow.com/training-a-tensorflow-object-detection-model-with-a-customdataset/
} 


\subsubsection{Results on THDD}

Classification evaluation: Fig. 6 shows the roc curves of the different CNNs. ical region. CNNAFD-GoogLeNet presented precisely the maximum accuracy as illustrated in Fig. 15. On the other hand, it is very obvious that the accuracy of MobileNetV2 was almost equal to that of CNNAFD. Thus, it could be concluded that better results came from the fusion of the two networks.

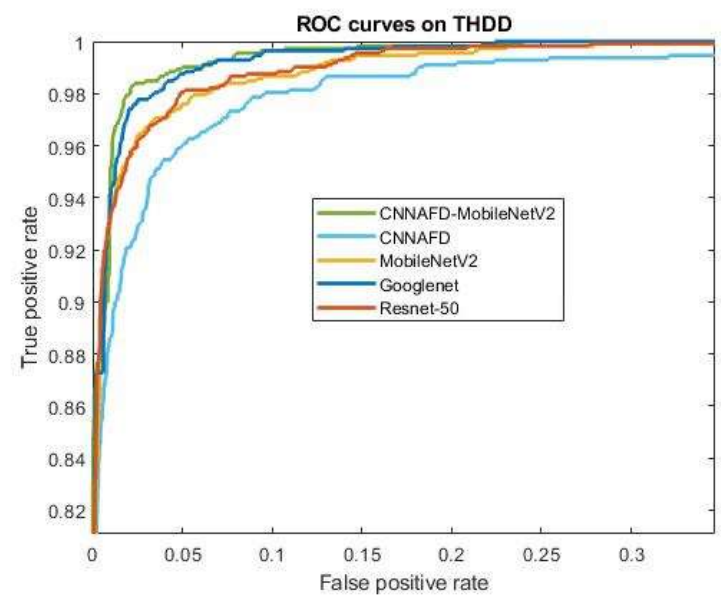

Figure 6: Comparison of ROC curves on THDD

Detection evaluation: Animals and mainly horses have many face texture variations that led to more difficulties in detection. Table 2 shows that our detector was better than the other detectors and achieved a higher performance. Fig. 7 displays some detection examples while Fig. 8 which represents the performance of our detector reports that the new detector had a big critical region.

\subsubsection{Results on Cat Database}

Classification evaluation: Fig. 9 shows the roc curves. CNNAFD-MobileNetV2 exhibited excellent results and outperformed all the other CNNs with the biggest critical region. In Fig. 15, CNNAFD-MobileNetV2 presents the best classifica- 
Table 2: A comparative study of horse face detection on THDD

\begin{tabular}{|l|l|l|l|l|}
\hline Method & AP & Recall & Precision & F1 \\
\hline Viola-Jones [37] & $70 \%$ & $40.12 \%$ & $73.00 \%$ & $51.78 \%$ \\
\hline SSD (MobileNetV2) [36] & $50.00 \%$ & - & - & - \\
\hline Detectron2 (ResNext-101) [33] & $98.89 \%$ & $99.60 \%$ & $88.24 \%$ & $93.57 \%$ \\
\hline YOLOv5 (CSPNet) [34] & $99.49 \%$ & $99.51 \%$ & $97.82 \%$ & $98.65 \%$ \\
\hline YOLOv3 (DarkNet-53) [31] & $98.13 \%$ & $98.55 \%$ & $99.51 \%$ & $99.02 \%$ \\
\hline YOLOv2 (MobileNetV2) & $97.81 \%$ & $97.83 \%$ & $99.75 \%$ & $98.78 \%$ \\
\hline $\begin{array}{l}\text { YOLOv2 } \\
\text { (MobileNetV2+CNNAFD) }\end{array}$ & $\mathbf{9 8 . 2 8 \%}$ & $\mathbf{9 8 . 3 1 \%}$ & $\mathbf{9 9 . 2 7 \%}$ & $\mathbf{9 8 . 7 9 \%}$ \\
\hline
\end{tabular}
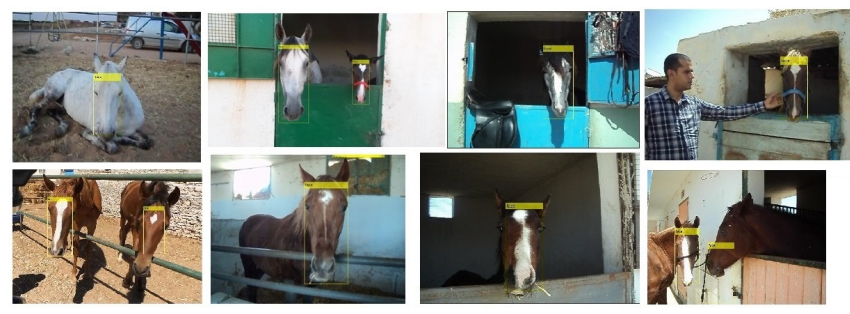

Figure 7: An illustration of horse face detection results

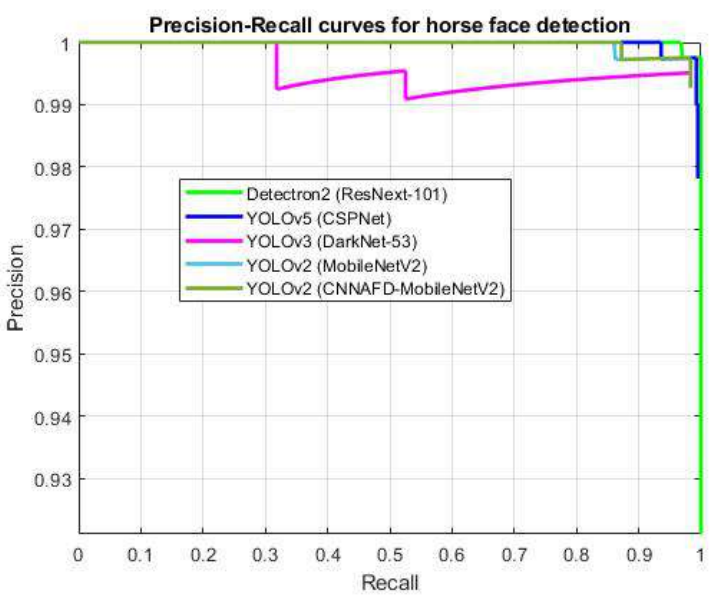

Figure 8: Precision-recall curves on THDD 
very large compared to the accuracy of CNNAFD. Therefore, it could not be concluded that one of the two networks was better than the other according to the horse and cat face results. Thus, it could be stated that the fusion of the two networks led to cooperation between them.

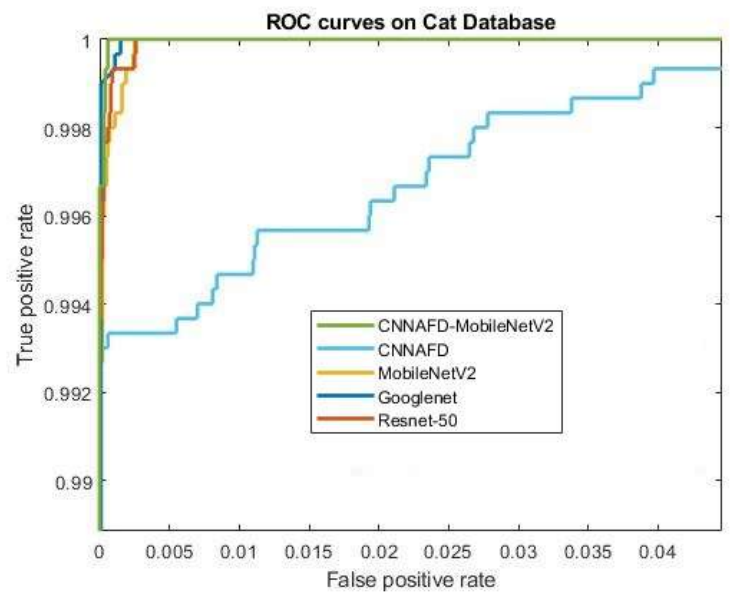

Figure 9: Comparison of ROC curves on Cat Database

Detection evaluation: The proposed detector was evaluated on the challenging Cat Database. Newly-published methods were compared to our results as illustrated in table 3. It was suggested that the same database partition of the related work could be taken. 5000 randomly-chosen images were used for training and 3000 ones for testing. CNNAFD-MobileNetV2 achieved a superior performance compared with the other approaches by a recall rate of $99.80 \%$ and a precision rate of $99.53 \%$. Fig. 10 shows the precision-recall curves on the Cat Database. Our detector presented the biggest critical region. Fig. 11, however, displays some detection examples. The performance of our detector was very apparent.

\subsubsection{Results on Stanford Dogs Dataset}

Classification evaluation: Fig. 12 shows the roc curves. CNNAFD-MobileNetV2 outperformed all the other CNNs with the biggest critical region. In precise detail, CNNAFD-MobileNetV2 presented the best classification accuracy as il- 


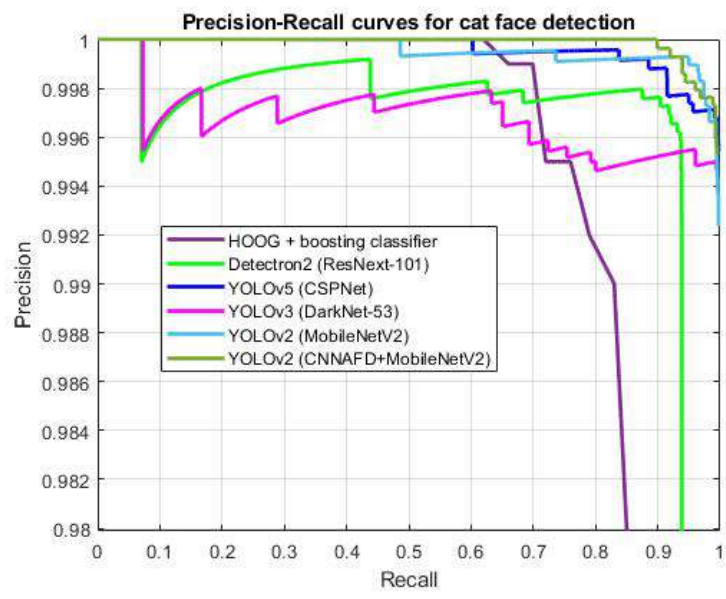

Figure 10: Comparison of Precision-recall curves on Cat Database

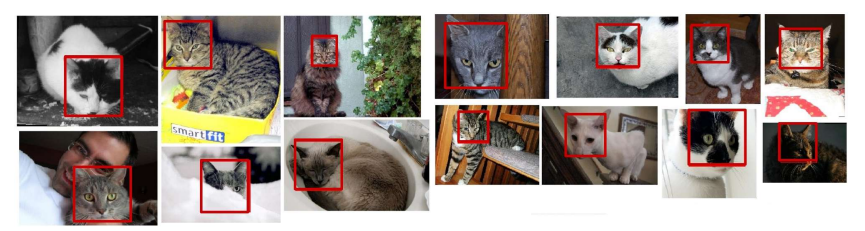

Figure 11: An illustration of cat face detection

lustrated in Fig. 15 Nevertheless, it is very noticeable that the accuracy of CNNAFD was large compared to that of MobileNetV2. In fact, it is obvious that the CNNAFD defeated MobileNetV2 in image classification on the Stanford Dogs Dataset. However, MobileNetV2 was second to none compared with CNNAFD on Cat Database. Hence, we conclude that the two networks were complementary since the fusion reinforced this coherence.

Detection evaluation: The proposed detector on the challenging Stanford Dogs Dataset was evaluated (Fig. 13). Taking 3000 randomly chosen images for training and 100 images (as in the related work) for testing has been suggested in our work. Our detector achieved a recall rate of $99 \%$ and a precision rate of 99.98\%, outperforming all recently detection methods (table 47. Fig. 14 shows a comparison between CNNAFD-MobileNetV2 and the related work detectors using recall-precision curves. The CNNAFD-MobileNetV2 presented the biggest 
Table 3: A comparative study of cat face detection on Cat Database

\begin{tabular}{|l|l|l|l|l|}
\hline Method & AP & Recall & Precision & F1 \\
\hline HOOG + boosting classifier [7] & - & $99,80 \%$ & $95.00 \%$ & $97,34 \%$ \\
\hline $\begin{array}{l}\text { Edge features and contrast } \\
\text { + M-L Classifier [8] }\end{array}$ & - & $85.00 \%$ & - & - \\
\hline $\begin{array}{l}\text { HAAR and HOG features } \\
\text { + Cascade classifiers [9] }\end{array}$ & - & $96.60 \%$ & $75.70 \%$ & $84.88 \%$ \\
\hline HAAR-cascade Detection [38] & $74.00 \%$ & $73,86 \%$ & $80,36 \%$ & $76,97 \%$ \\
\hline HAAR-cascade Detection [39] & $74.00 \%$ & $75.34 \%$ & $85.94 \%$ & $80.29 \%$ \\
\hline SSD (MobileNetV2) [36] & $63.00 \%$ & - & - & - \\
\hline Detectron2 (ResNext-101) [33] & $93.70 \%$ & $93.88 \%$ & $97.63 \%$ & $95.71 \%$ \\
\hline YOLOv5 (CSPNet) [34] & $99.71 \%$ & $99.75 \%$ & $99.54 \%$ & $99.64 \%$ \\
\hline YOLOv3 (DarkNet-53) [31] & $99.31 \%$ & $99.63 \%$ & $99.47 \%$ & $99.54 \%$ \\
\hline YOLOv2 (MobileNetV2) & $99.72 \%$ & $99.77 \%$ & $99.24 \%$ & $99.50 \%$ \\
\hline $\begin{array}{l}\text { YOLOv2 } \\
\text { (MobileNetV2+CNNAFD) }\end{array}$ & $\mathbf{9 9 . 7 8 \%}$ & $\mathbf{9 9 . 8 0 \%}$ & $\mathbf{9 9 . 5 3 \%}$ & $\mathbf{9 9 . 6 6 \%}$ \\
\hline
\end{tabular}

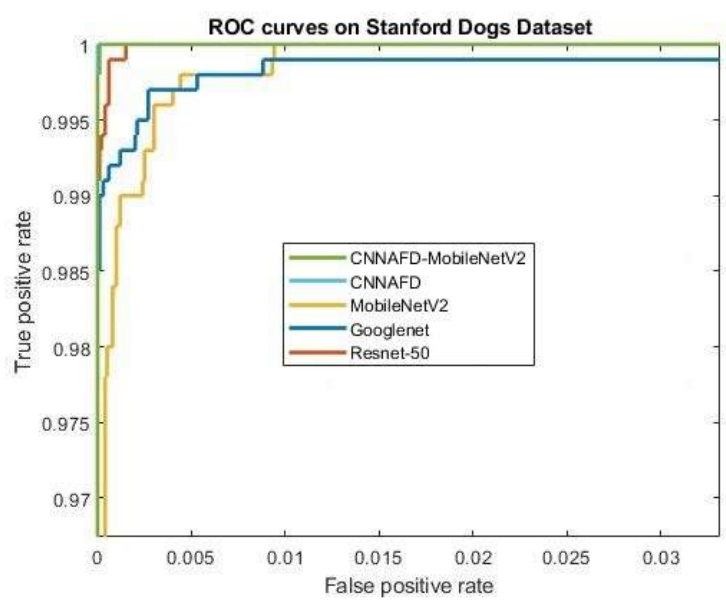

Figure 12: Comparison of ROC curves on Stanford Dogs Dataset 


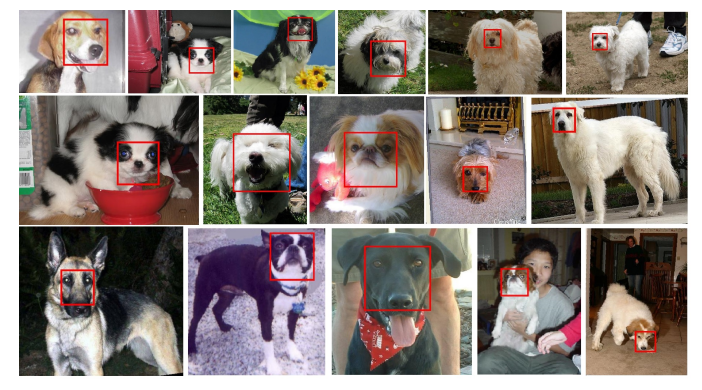

Figure 13: An illustration of dog face detection results

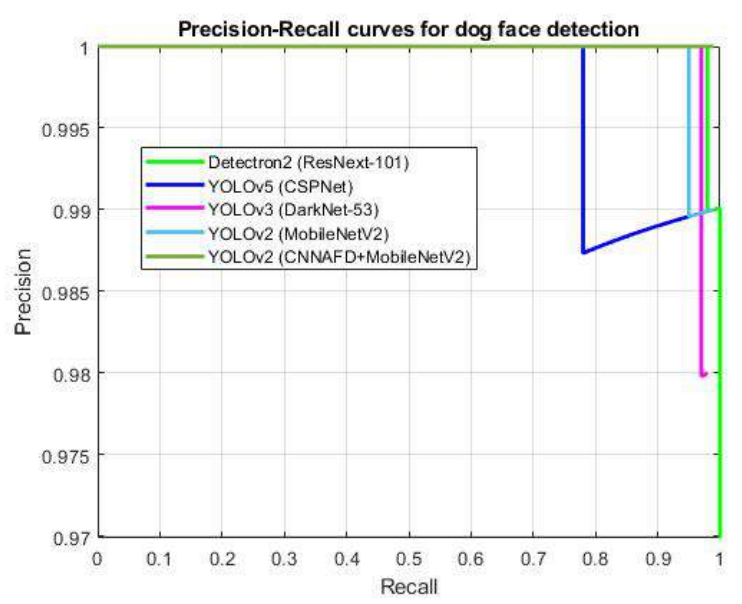

Figure 14: Comparison of precision-recall curves on Stanford Dogs Dataset

critical region.

\section{Conclusion}

In this paper, we proposed the CNNAFD for Animal Face Detection (horses, cats and dogs). We demonstrated the performance of the proposed CNNAFD network on three real-world databases. The proposed sparse ANOFS-CONV layer and the proposed training methodology contributed to distinguish properly between true and false detections (face/non-face). CNNAFD extracted the relevant features using ANOF-CONV layer and then classified the candidate 285 bloc using the stacked Fully Connected layers. For more efficiency, the addition 
Table 4: A comparative study of dog face detection

\begin{tabular}{|l|l|l|l|l|}
\hline Approach & AP & Recall & Precision & F1 \\
\hline $\begin{array}{l}\text { Edge features and contrast } \\
+ \text { M-L Classifier [8] }\end{array}$ & - & $90.00 \%$ & - & - \\
\hline $\begin{array}{l}\text { HAAR and HOG features } \\
\text { + Cascading classifiers [9] }\end{array}$ & - & $98.30 \%$ & $90.80 \%$ & $94.40 \%$ \\
\hline SSD (MobileNetV2) [36] & $79.00 \%$ & - & - & - \\
\hline Detectron2 (ResNext-101) [33] & $98.53 \%$ & $99.00 \%$ & $93.46 \%$ & $96.15 \%$ \\
\hline YOLOv5 (CSPNet) [34] & $97.98 \%$ & $98.00 \%$ & $98.99 \%$ & $98.49 \%$ \\
\hline YOLOv3 (DarkNet-53) [31] & $97.98 \%$ & $98.00 \%$ & $98.00 \%$ & $98.00 \%$ \\
\hline YOLOv2 (MobileNetV2) & $98.96 \%$ & $99.00 \%$ & $99.00 \%$ & $99.00 \%$ \\
\hline $\begin{array}{l}\text { YOLOv2 } \\
\text { (MobileNetV2 +CNNAFD) }\end{array}$ & $\mathbf{9 9 . 0 0 \%}$ & $\mathbf{9 9 . 0 0 \%}$ & $\mathbf{9 9 . 9 8 \%}$ & $\mathbf{9 9 . 4 9 \%}$ \\
\hline
\end{tabular}

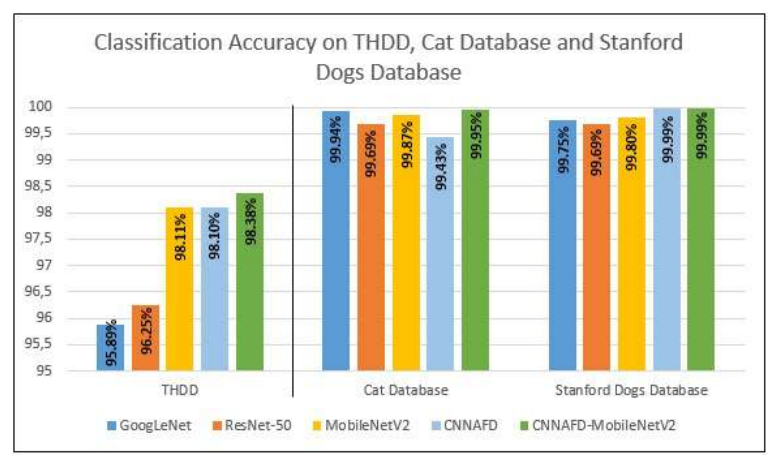

Figure 15: Classification results using GoogleNet, ResNet-50, MobileNetV2, CNNAFD and CNNAFD-MobileNetV2 
of CNNAFD was proposed on the YOLOv2 detector with MobileNetV2 to reinforce the animal face detection process. The resulting decisions of MobileNetV2 and CNNAFD were merged by the final FC layer to obtain the final detection decision. In fact, the fusion of CNNAFD with the MobileNetV2 helped to increase the precision rate and to decrease the number of false detections. The proposed detector using the CNNAFD-MobileNetV2 backbone outperformed the state of the art detectors and gave an average precision equal to $98,28 \%$, $99.78 \%$ and $99 \%$ for horses, cats and dogs respectively.

As futures work, we aim to improve the performance of CNNAFD-MobileNetV2 by exploring more discriminant filters. Also, we intend to extend our proposed detector to other animals and to apply it for human face detection.

\section{References}

[1] C. Zhang, Z. Zhang, A survey of recent advances in face detection, Tech. Rep. MSR-TR-2010-66 (June 2010).

[2] P. Taunk, G. Jayasri, P. Priya J, N. S. Kumar, Face detection using viola jones with haar cascade, in: Test Engineering and Management, Vol. 83, 2020 .

[3] B. Zhang, J. Li, Y. Wang, Y. Tai, C. Wang, J. Li, F. Huang, Y. Xia, W. Pei, R. Ji, Asfd: Automatic and scalable face detector, in: arXiv, 2020.

[4] J. Duan, S. Liao, S. Zhou, S. Z.Li, Face classification: A specialized benchmark study, in: Chinese Conference on Biometric Recognition, Vol. 9967, 2016, pp. 22-29. doi:10.1007/978-3-319-46654-5_3.

[5] S. Joshi, D. K. Verma, G. Saxena, A. Paraye, Issues in training a convolutional neural network model for image classification, in: International Conference on Advances in Computing and Data Sciences, Vol. 1046, 2019, pp. 282-293. doi:10.1007/978-981-13-9942-8_27. 
[6] S. Sabour, N. Frosst, G. Hinton, Dynamic routing between capsules, in: NIPS'17: Proceedings of the 31st International Conference on Neural Information Processing Systems, 2017, p. 3859-3869.

[7] W. Zhang, J. Sun, X. Tang, From tiger to panda: Animal head detection, in: 2011 IEEE Transactions on Image Processing, Vol. 20, 2011, pp. 16961708.

[8] A. Yamada, K. Kojima, J. Kiyama, M. Okamoto, H. Murata, Directional edge-based dog and cat face detection method for digital camera, in: IEEE International Conference on Consumer Electronics (ICCE), 2011, pp. 8788. doi:10.1109/ICCE.2011.5722916.

[9] N. Mukai, Y. Zhang, Y. Chang, Pet face detection, in: 2018 Nicograph

International (NicoInt), 2018, pp. 52-57. doi:10.1109/NICOINT.2018. 00018

325 [10] A. Vlachynska, Z. K. Oplatkova, T. Turecek, Dog face detection and localization of dogface's landmarks, in: Silhavy R. (eds) Artificial Intelligence and Algorithms in Intelligent Systems. CSOC2018, Vol. 764, 2018, pp. 465476. doi:10.1007/978-3-319-91189-2_46.

[11] A. Tureckova, T. Holik, Z. K. Oplatkova, Dog face detection using yolo network, in: MENDEL Soft Computing Journal, Vol. 26, 2020, pp. 25713701. doi:10.13164/mendel.2020.2.017.

[12] I. Jarraya, W. Ouarda, A. M. Alimi, A preliminary investigation on horses recognition using facial texture features, in: 2015 IEEE International Conference on Systems, Man, and Cybernetics, 2015, pp. 2803-2808. doi:10.1109/SMC.2015.489

[13] W. Ouarda, H. Trichili, A. M. ALIMI, B. Solaiman, Towards a novel biometric system for smart riding club, Journal of information assurance and security (JIAS) 11 (2016) $201-213$. 
[14] I. Jarraya, W. Ouarda, A. M. Alimi, Deep neural network features for horses identity recognition using multiview horses' face pattern, in: ICMV, Vol. 10341, 2017, pp. 52 - 56. doi:10.1117/12.2269064.

[15] N. Dalal, B. Triggs, Histograms of oriented gradients for human detection, in: 2005 IEEE Computer Society Conference on Computer Vision and Pattern Recognition (CVPR'05), Vol. 1, 2005, pp. 886-893 vol. 1. doi: 10.1109/CVPR.2005.177.

[16] F. Ben Said, A. M. Alimi, Anofs: Automated negotiation based online feature selection method, in: 2015 15th International Conference on Intelligent Systems Design and Applications (ISDA), 2015, pp. 225-230. doi:10.1109/ISDA.2015.7489229.

[17] F. B. Said, A. M. Alimi, Multi agent-learner based online feature selection system, in: 2016 IEEE International Conference on Systems, Man, and Cybernetics (SMC), 2016, pp. 003652-003657. doi:10.1109/SMC.2016. 7844801 .

[18] F. BenSaid, A. M. Alimi, Online feature selection system for 355 big data classification based on multi-objective automated ne1 gotiation, Pattern Recognition 110 (2021) 107629. doi:https: //doi.org/10.1016/j.patcog.2020.107629.

1 URL https://www.sciencedirect.com/science/article/pii/ S0031320320304325

[19] A. Krizhevsky, I. Sutskever, G. E. Hinton, Imagenet classification with deep convolutional neural networks, Vol. 25, 2012. doi:10.1145/3065386

[20] K. Simonyan, A. Zisserman, Very deep convolutional networks for largescale image recognition, in: Y. Bengio, Y. LeCun (Eds.), 3rd International Conference on Learning Representations, ICLR 2015, San Diego, CA, USA, May 7-9, 2015, Conference Track Proceedings, 2015. 
[21] C. Szegedy, V. Vanhoucke, S. Ioffe, J. Shlens, Z. Wojna, Rethinking the inception architecture for computer vision, in: ArXiv, 2015.

[22] C. Szegedy, V. Vanhoucke, S. Ioffe, J. Shlens, Z. Wojna, Rethinking the inception architecture for computer vision, in: 2016 IEEE Conference on Computer Vision and Pattern Recognition (CVPR), 2016, pp. 2818-2826. doi:10.1109/CVPR.2016.308

[23] S. Zagoruyko, N. Komodakis, Wide residual networks, in: ArXiv, 2017.

[24] G. Huang, Z. Liu, L. Van Der Maaten, K. Q. Weinberger, Densely connected convolutional networks, in: 2017 IEEE Conference on Computer Vision and Pattern Recognition (CVPR), 2017, pp. 2261-2269. doi: 10.1109/CVPR.2017.243.

[25] M. Sandler, A. Howard, M. Zhu, A. Zhmoginov, L. Chen, Mobilenetv2: Inverted residuals and linear bottlenecks, in: 2018 IEEE/CVF Conference on Computer Vision and Pattern Recognition, 2018, pp. 4510-4520. doi: 10.1109/CVPR.2018.00474

[26] T. Korte, 10,000 cat pictures (for science), Center for data innovation.

[27] A. Khosla, N. Jayadevaprakash, B. Yao, F.-F. Li, L.: Novel dataset for finegrained image categorization, in: First Workshop on Fine-Grained Visual Categorization, CVPR, 2012.

[28] W. Liu, I. Hasan, S. Liao, Center and scale prediction: A box-free approach for pedestrian and face detection, in: arXiv: Computer Vision and Pattern Recognition, 2019.

[29] B. Zhang, J. Li, Y. Wang, Y. Tai, C. Wang, J. Li, F. Huang, Y. Xia, W. Pei, R. Ji, Asfd: Automatic and scalable face detector, in: arXiv: Computer Vision and Pattern Recognition, 2020.

[30] S. Ren, K. He, R. Girshick, J. Sun, Faster r-cnn: Towards real-time object detection with region proposal networks, in: C. Cortes, N. Lawrence, 
D. Lee, M. Sugiyama, R. Garnett (Eds.), Advances in Neural Information Processing Systems, Vol. 28, Curran Associates, Inc., 2015, pp. 91-99.

395

[31] J. Redmon, A. Farhadi, Yolov3: An incremental improvement, in: ArXiv: Computer Vision and Pattern Recognition, 2018.

[32] W. Liu, D. Anguelov, D. Erhan, C. Szegedy, S. Reed, C.-Y. Fu, A. C. Berg, Ssd: Single shot multibox detector, European Conference on Computer Vision 9905 (2016) 21-37.

[33] Y. Wu, A. Kirillov, F. Massa, W.-Y. Lo, R. Girshick, Detectron2, https: //github.com/facebookresearch/detectron2 (2019).

[34] G. Jocher, A. Stoken, J. Borovec, NanoCode012, ChristopherSTAN, L. Changyu, Laughing, A. Hogan, lorenzomammana, tkianai, yxNONG, AlexWang1900, L. Diaconu, Marc, wanghaoyang0106, ml5ah, Doug, Hatovix, J. Poznanski, L. Yu, changyu98, P. Rai, R. Ferriday, T. Sullivan, W. Xinyu, YuriRibeiro, R. Eduard Claramunt, hopesala, p. dave, yzchen, ultralytics/yolov5: v3.0, Tech. rep. (2020). doi:10.5281/zenodo.3983579. URL https://zenodo.org/record/3983579\#.YKUgT6HjLIU

[35] C.-Y. Wang, H.-Y. M. Liao, I.-H. Yeh, Y.-H. Wu, P.-Y. Chen, J.-W. Hsieh, Cspnet: A new backbone that can enhance learning capability of cnn, in: ArXiv: Computer Vision and Pattern Recognition, 2019. doi:arXiv:1911.11929.

[36] J. Nelson, Training a tensorflow mobilenet object detection model with a custom dataset, Tech. rep. (02 2020).

[37] V. Paul, M. J. Jones, Robust real-time face detection, in: International

a Journal of Computer Vision, Vol. 57, 2004, p. 137-154. doi:10.1023/B: VISI.0000013087.49260.fb

[38] OpenCV, Haarcascade frontal cat face, Tech. rep. (2018).

[39] OpenCV, Haarcascade frontal cat face extended, Tech. rep. (2018). 